The TOTEM T1 read out card motherboard

This article has been downloaded from IOPscience. Please scroll down to see the full text article. 2010 JINST 5 C12052

(http://iopscience.iop.org/1748-0221/5/12/C12052)

View the table of contents for this issue, or go to the journal homepage for more

Download details:

IP Address: 137.138.124.142

The article was downloaded on 11/07/2011 at 12:43

Please note that terms and conditions apply. 
Topical Workshop on Electronics for Particle Physics 2010 , 20-24 SEPTEMBER 2010, AACHEN, GERMANY

\title{
The TOTEM T1 read out card motherboard
}

\author{
S. Minutoli, ${ }^{a, 1}$ M. Lo Vetere ${ }^{a, b}$ and E. Robutti ${ }^{a}$ \\ ${ }^{a}$ Istituto Nazionale di Fisica Nucleare, \\ Via Dodecaneso 33, 16146 Genoa, Italy \\ ${ }^{b}$ University of Genova - Department of Physics, \\ Via Dodecaneso 33, 16146 Genoa, Italy \\ E-mail: Saverio.Minutoli@ge.infn.it
}

ABSTRACT: This article describes the Read Out Card (ROC) motherboard, which is the main component of the T1 forward telescope front-end electronic system. The ROC main objectives are to acquire tracking data and trigger information from the detector. It performs data conversion from electrical to optical format and transfers the data streams to the next level of the system and it implements Slow Control modules which are able to receive, decode and distribute the LHC machine low jitter clock and fast command. The ROC also provides a spy mezzanine connection based on programmable FPGA and USB2.0 for laboratory and portable DAQ debugging system.

KEYWORDS: Optical detector readout concepts; Data acquisition circuits; Front-end electronics for detector readout; Digital electronic circuits

\footnotetext{
${ }^{1}$ Corresponding author.
} 


\section{Contents}

1 Introduction 1

2 General design specifications 2

3 The Read Out Card motherboard 3

3.1 Tracking data conversion and transmission 3

3.2 Trigger data transmission 4

3.3 Clock and fast commands 4

3.4 Slow control ring 5

3.5 Auxiliary debugging port 5

3.6 Low voltage distribution 5

\section{Introduction}

The TOTEM experiment [1] is designed to measure the proton-proton elastic and total cross section at the CERN Large Hadron Collider (LHC) and is composed of three sub-detector telescopes: T1 equipped with Cathode Strip Chambers (CSC), T2 with Gas Electron Multiplier detectors (GEM) and Roman Pots with silicon edgeless microstrip silicon detector. The T1 detector will measure the inelastic rate in the pseudo-rapidity region $3.1 \leqslant|\eta| \leqslant 4.7$.

The T1 telescope consists of four sets (quarters) of five equally spaced planes of CSC detectors which in turn generate about 11000 anodes (AFECs) and 16000 cathodes (CFECs) signals. Data from each of these must be processed and optically transmitted to the counting room.

A basic block diagram of the functional components of one quarter of the system is shown in figure 1. Three logically and physically separated regions make up the T1 electronic system [2]: the "On Detector Region" where the Front-End ASICs are located, the "Local Detector Region" where the data are collected and optically transmitted via the Read-Out Card motherboards (ROC) to the "Counting Room Region" where they are received by the TOTEM Front End Driver (TOTFED).

The charge readout of the detectors is based on the TOTEM front-end ASIC named VFAT [3]. The chip contains both analog and digital functions traditionally found on separate components. The VFAT is a very low noise 128 channels front-end amplifier with programmable internal calibration, intelligent "fast OR" trigger outputs, digital data tagging and storage, data formatting and data packet transmission with error protection.

Up to 16 VFATs, mounted on custom hybrids, are connected to each of the 36 ROC ( 9 per T1 quarter) via flexible connections. 


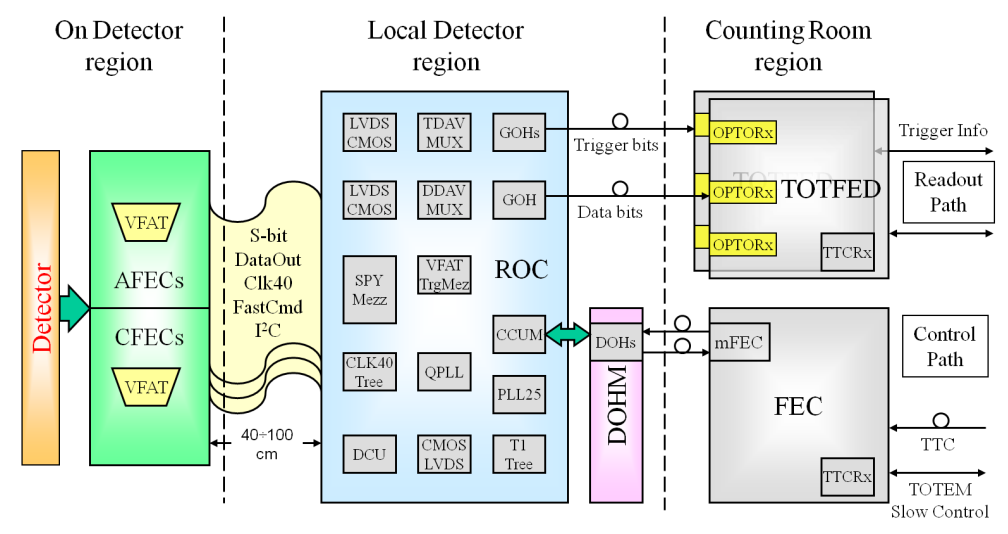

Figure 1. T1 electronic system architecture.

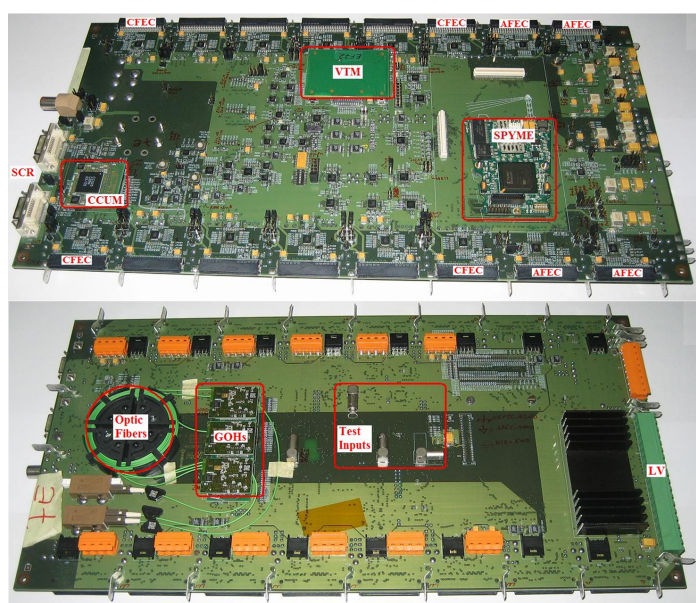

Figure 2. ROC motherboard - top and bottom side.

\section{General design specifications}

The ROC which is the electric junction point of two CSC detectors must fit in the T1 telescope complex mechanics. It connects up to 16 hybrids on the anodes and cathodes cards and finally controls 2048 signals to and from the outside world. The connections to the detector ASICs are made by flexible shielded fine pitch flat cables while the other connections are performed by twisted pairs ribbon cables and single mode ribbon optic fibers.

The layout of the ROC, given the large number of connections and other size limitations, results in a very challenging 12 layers layout.

Besides the electrical functionality described in detail in the following, the design of the ROC is constrained by mechanics and radiation tolerance considerations.

In order to guarantee robustness to the large radiation doses the ROC will be exposed to, all the active components used in the ROC are design radiation tolerant components fabricated in the standard $0.25 \mu \mathrm{m}$ CMOS technology and employing radiation tolerant layout practices $[4,5]$. Safety regulations imposed also fabrication of the board with halogen free material.

Figure 2 shows the two sides of the completed board with mezzanine cards mounted. The black connectors (top) placed along the long sides of the board are for detector signal connection; on the 


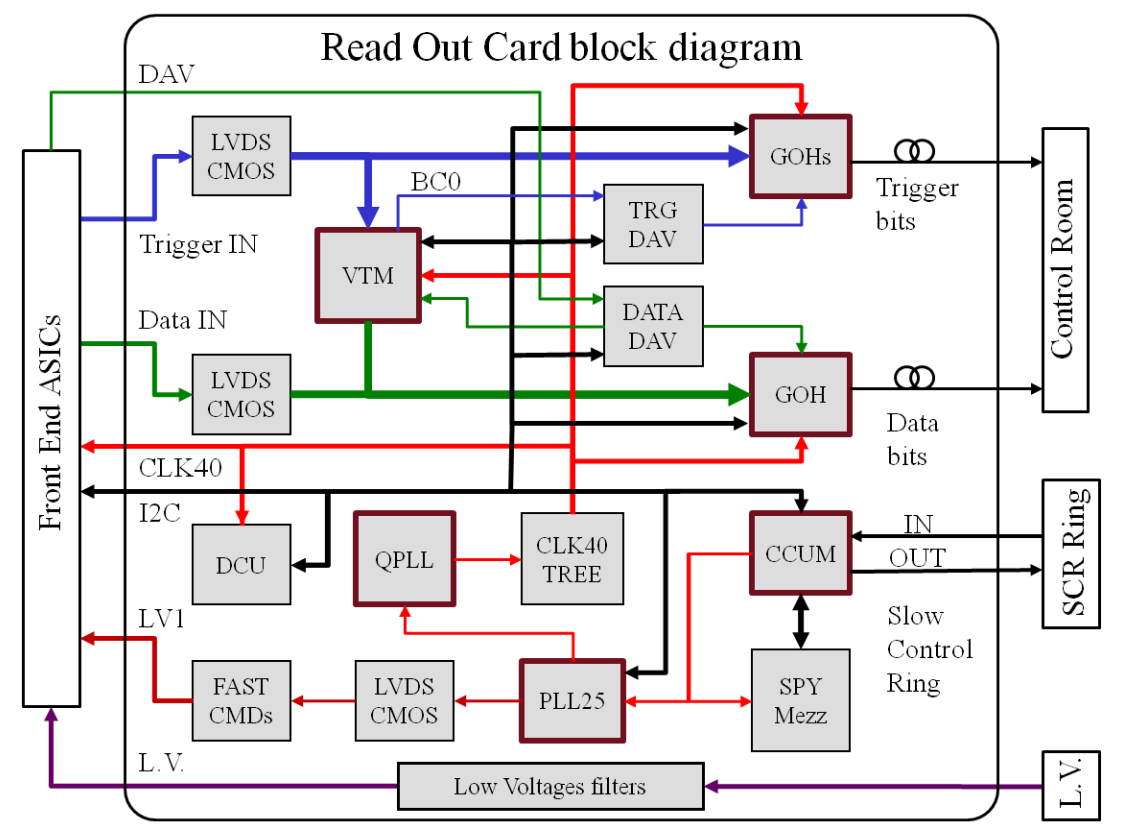

Figure 3. ROC motherboard block diagram.

short sides one can see on the left the connectors for the Slow Control Ring (top), Tracking and Trigger Data (bottom). The Low Voltage connectors are on the bottom side on the right. Grounding and shielding connections can be made via several lugs close to the detector signal input connectors and on the metallic cover of the ROC (not shown in the figure).

\section{The Read Out Card motherboard}

The ROC motherboard functional block diagram is shown in figure 3. The blocks are described in detail below:

\subsection{Tracking data conversion and transmission}

This block is based on LVDS to CMOS converters and Gigabit Optical Hybrid (GOH) [6] module. The GOH performs data conversion from electrical to optical format and transfers the data streams to the next level of the system over an optic fiber through a $0.8-1.6 \mathrm{Gbit} / \mathrm{s}$ Ethernet $8 \mathrm{~B} / 10 \mathrm{~B}$ parallelto-serial encoder. One GOH mezzanine (figure 4) is used to send data from 16 VFAT hybrids to the Counting Room (CR). The GOH transmission, driven by the DAta Valid (DAV) signal generated by the master ${ }^{1}$ VFAT, is enabled through MUX circuitry programmable via Slow Control Ring (SCR) [7].

The "Hit" information is coded in the form of binary channel data corresponding to the clock period selected by a first level trigger (LV1A) signal. On receipt of a trigger (generated after a given latency period), the binary data, the time stamps and other information (i.e. ChipID, CRC) are packed into a 192-bit data packet.

\footnotetext{
${ }^{1}$ The master DAV can be selected from four available sources, respectively the first anode and the first cathode VFAT of each of the 2 CSC chambers managed by the ROC.
} 


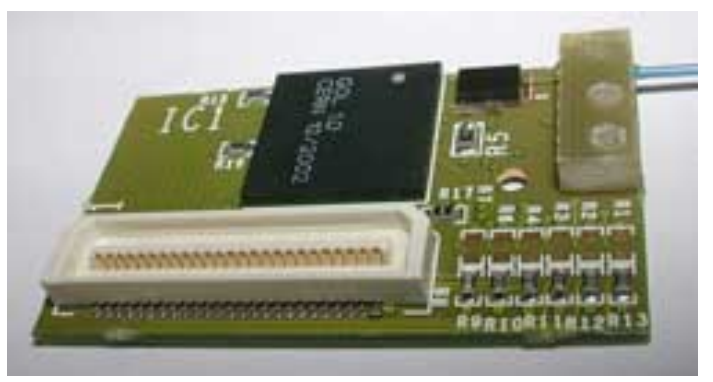

Figure 4. GOH mezzanine.

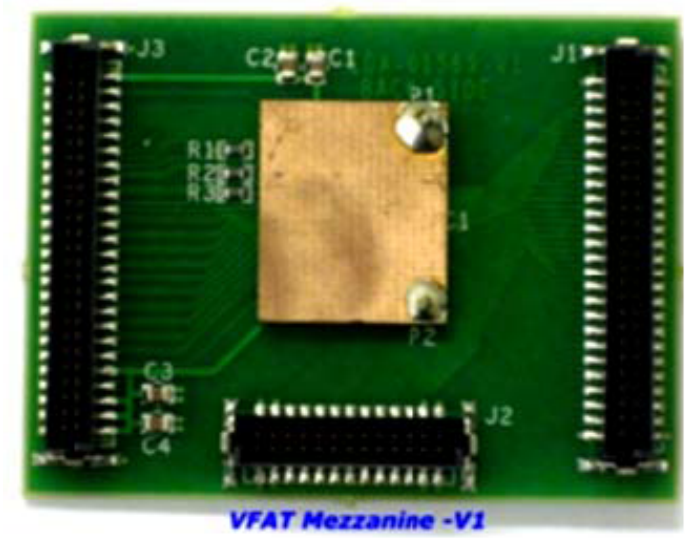

Figure 5. VFAT trigger mezzanine.

\subsection{Trigger data transmission}

This block is based on LVDS to CMOS converters, VFAT Trigger Mezzanine (VTM, figure 5) and two GOH modules for the optical transmission to the CR. The GOH transmission can be enabled via SCR and sourced by several devices.

Since the trigger signals are sent every clock cycle a time reference has to be included in the trigger data stream to facilitate recovering the correspondence between the event and the transmitted bits. This is done from the fast command Bunch Crossing 0 (BC0) by the VTM. The VFAT on board decodes the BC0 signal and provides this to a circuit which disables the GOH's DAV signal upon reception of the $\mathrm{BC} 0$ for the duration of one clock cycle. This will be recognized in the counting room providing the time reference.

\subsection{Clock and fast commands}

This block is based on a PLL25 chip [8], a QPLL and a number of clock and command distribution circuits. It provides synchronous clock and commands to each component on the board including the VFAT chips on the FE hybrids. It extracts and distributes a three bits coded fast command (LV1, $\mathrm{BC} 0$, Calib, Resync) from the LHC clock main system and set a programmable latency period (up to $6.4 \mu \mathrm{s}$ ). The circuitry allows the equalization of the clock timing for the anode and cathode hybrids which are connected with different cables length. 


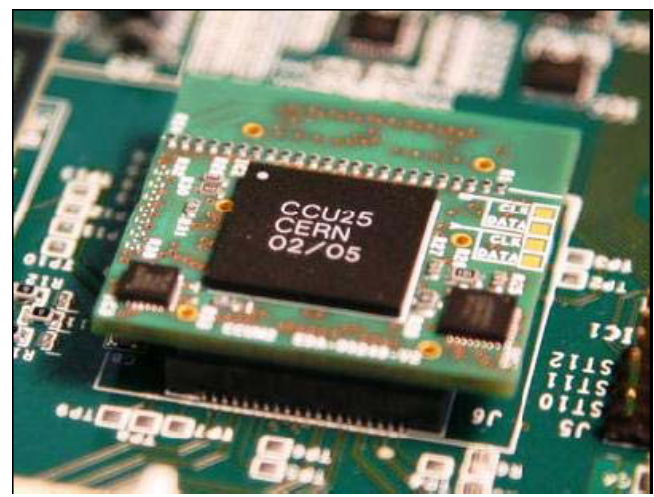

Figure 6. CCUM mezzanine.

\subsection{Slow control ring}

This block is based on CERN Communication and Control Unit CCUM mezzanine [7] (figure 6). This module is connected to the control loop ${ }^{2}$ (DOHM - FEC) via two 20 pins $3 \mathrm{M}$ high speed connectors. The control logic block provides $16 \mathrm{I}^{2} \mathrm{C}$ interface channels and one 8 bit bidirectional parallel control port.

All the system parameters (i.e. FE hybrid values, clock latency) are sent to the devices via $\mathrm{I}^{2} \mathrm{C}$, while GOHs power cycle, GOHs status, Data and Trigger GOH sources, Soft and Hard Reset are managed via the parallel port. A skip fault architecture for additional redundancy based on the doubling of signal paths and the possibility of bypassing interconnection lines between CCUM is also implemented.

\subsection{Auxiliary debugging port}

A spy test port is foreseen on the ROC board: all the front-end connections are routed to dedicated connectors to which one may plug a piggy-back programmable custom mezzanine (SPYME) equipped with a Xilinx FPGA type XC3S1500FG456. The FPGA emulates the DAQ system located in the CR and allows to remotely test all the ROC functionalities via a USB 2.0 port.

\subsection{Low voltage distribution}

The ROC needs to receive low voltage power at $2.5 \mathrm{~V}$ for its own operation, and for the operation of the hybrids. Independent PCB layers are used for the analog and digital low voltages distribution to the hybrids, both powered at $2.5 \mathrm{~V}$. The total currents drawn are respectively $1.6 \mathrm{~A}$ and $3.3 \mathrm{~A}$ for the analog and digital voltages.

\section{Acknowledgments}

The authors would like to thank Dr. Fabrizio Ferro and Prof. Marco Bozzo for their valuable contribution during the ROC motherboard debugging phase and integration inside the T1 electronic system and Stefano Cerchi for its contribution during the assembling of the ROC on the T1 mechanical structure.

\footnotetext{
${ }^{2}$ The TOTEM slow control system uses a ring topology configured as a local area network. The connections between ROCs are done electrically using differential lines (LVDS), the first and last CCUM of the chain are optically converted and connected to the master controller in the Counting Room.
} 


\section{References}

[1] TOTEM collaboration, G. Anelli et al., The TOTEM experiment at the CERN Large Hadron Collider, 2008 JINST 3 S08007.

[2] S. Minutoli et al., The digital read-out for the CSC system of the TOTEM experiment at LHC, Nucl. Instrum. Meth. A 617 (2010) 342.

[3] P. Aspell, et al., VFAT2: a front-end system on chip providing fast trigger information, digitized data storage and formatting for the charge sensitive readout of multi channel silicon and gas particle detectors, in the proceedings of Topical Workshop on Electronics for Particle Physics 2007 (TWEPP-07), September 3-7, Prague, Czech Republic (2007).

[4] G. Anelli et al., Radiation tolerant VLSI circuits in standard deep submicron CMOS technologies: practical design aspects, IEEE Trans. Nucl.Sci. 46 (1999) 1690.

[5] F. Faccio et al., Total dose and single event effects (SEE) in a $0.25 \mu \mathrm{m}$ technology, in the proceedings of the $4^{\text {th }}$ Workshop on Electronics for LHC experiments, September 21-25, Rome, Italy (1998), p. 105.

[6] P. Moreira et al., A radiation tolerant gigabit serializer for LHC data transmission, talk given at the $7^{\text {th }}$ Workshop on Electronics for LHC Experiments, September 10-14, Stockholm, Sweden (2001).

[7] C. Paillard et al., The CCU25: a network oriented communication and control unit integrated circuit in a 0,25mm CMOS technology, talk given at the $8^{\text {th }}$ Workshop on Electronics for LHC Experiments, September 9-13, Colmar, France (2002).

[8] A. Marchioro et al., A PLL-delay ASIC for clock recovery and trigger distribution in the CMS tracker, talk given at the $3^{\text {rd }}$ Workshop on Electronics for LHC Experiments, September 22-26, London, U.K. (1997). 